\title{
Compartment Syndrome Following Lower Limb Arthroplasty: A Review
}

\author{
Nikolaos G. Lasanianos ${ }^{1}$, Nikolaos K. Kanakaris ${ }^{1}$, Craig S. Roberts ${ }^{2}$ and Peter V. Giannoudis ${ }^{* 1}$ \\ ${ }^{1}$ Academic Department of Trauma and Orthopaedics, School of Medicine, University of Leeds, Leeds General Infirmary, \\ Leeds, UK \\ ${ }^{2}$ Academic Department of Trauma and Orthopaedics, School of Medicine, University of Louisville, Kentucky, USA
}

\begin{abstract}
Compartment syndrome is an urgent clinical entity characterised by an increase in the interstitial pressure within a closed osseofascial compartment. Although well recognised as a potential complication after orthopaedic trauma, it is very rarely presented after elective orthopaedic surgery and especially joint arthroplasty. In these rare cases a number of variables are associated with it (positioning, coagulopathy, extensive soft tissue dissection, previous scarring, and epidural analgesia). In this study we present the current evidence with regard to incidence and causation of compartment syndrome after lower limb joint arthroplasty and make recommendations on how to avoid the development of this devastating complication.
\end{abstract}

Keywords: Hip, knee, arthroplasty, TKA, THA, compartment syndrome, review.

\section{INTRODUCTION}

Acute compartment syndrome (CS) of the lower extremity is a surgical emergency induced by bleeding, or oedema within a closed, non-elastic muscle compartment, leading to high intra-compartmental pressures compromising local tissue perfusion [1]. Prompt diagnosis is of paramount importance in order to avoid irreversible tissue changes leading to muscle necrosis, neurological deficit, myoglobinuria, renal failure, and potentially mortality.

The most common cause of CS is trauma, usually orthopaedic or vascular [2-4]. Other aetiologies that have been associated with the development of a CS, include exercise [5], positioning during prolonged surgical procedures [6], intramedullary nailing [7], prolonged tourniquet application [8], anticoagulation [9], and intravenous drug abuse [10]. CS has also been reported after elective orthopaedic procedures, mainly concerning total hip and knee arthroplasty. Following the gained popularity of lower limb arthroplasty [11], and the absence of an analysis of the resulting CS, the herein study aims to investigate the existing evidence on this complication in an effort to identify causative factors, and propose an algorithm of prevention and management.

\section{MATERIALS AND METHODOLOGY}

An electronic search of the MEDLINE database (up to December 2009) was carried out entering the terms and Boolean operators: "compartment syndrome" AND "arthroplasty". It has resulted to 29 studies. In addition, we reviewed the references from the resulting publications to identify further potential articles that could be included to our study. Review papers and editorials were excluded

*Address correspondence to this author at the Academic Department of Trauma \& Orthopaedics, Leeds General Infirmary, Clarendon wing, Level A, Great George Street, LS1 3EX, Leeds, UK; Tel: +44-113-3922750; Fax: 0044-113-3923290; E-mail: pgiannoudi@aol.com
(Table 1). All articles were screened with set inclusion and exclusion criteria.

Table 1. A Flowchart Illustrates our Study Selection Process

\section{REFERENCE DATABASES}

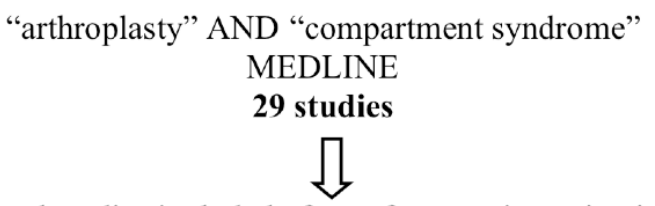

Additional studies included after references investigation $(+2)$<smiles>C1CCC(C2CCCC2)C1</smiles>

Exclusion of letters to the editors and reviews (-2)

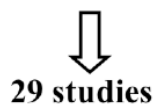

Exclusion of studies not fulfilling the inclusion criteria (-7)<smiles></smiles>

Exclusion of studies not available in the web as full text (-2)

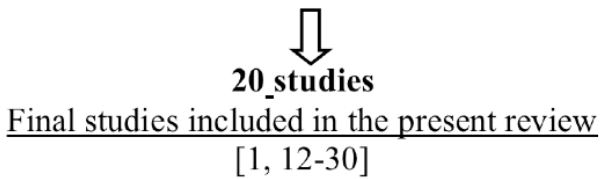

Articles were considered eligible if they met the following inclusion criteria: (1) a lower limb arthroplasty procedure should be described; (2) the symptoms or the complications described should correspond to a compartment syndrome. Exclusion criteria included studies that examined arthroplasty treatment in the setting of acute trauma, review 
articles, editorials, letters, and manuscripts in other than the English language.

Two separate reviewers (NGL, NKK) were involved in the screening process, and classified the retrieved studies independently, either as relevant or non-relevant to the present review. Any disagreements were resolved between the 2 reviewers after reading the manuscript's abstract.

Selected studies were original articles either in the form of case reports, or case series of lower limb joint arthroplasty in which CS was recorded between the complications. All relevant information regarding patients' age and gender, type of arthroplasty, reason for surgery, anatomical body region and involved compartments, positioning, use of tourniquet, bleeding diathesis, thromboprophylaxis administration, type of post-operative analgesia, operating time, time to discontinuation of epidural effusion, symptoms initiation and fasciotomy, neurovascular deficit, intracompartmental pressure (ICP) reading and complications were all recorded (Table 2).

\section{RESULTS}

In total twenty manuscripts met the eligibility criteria dating from 1979 to 2007 [1, 12-30] (Table 1). Thirteen studies consisted of a single case report $[12,13,15,16,18$ 22, 24-27], while 7 studies included small series of patients (2-7) either undergoing the same type of arthroplasty or developing a CS on the same site $[1,14,17,23,28-30]$. The first three chronological studies [28-30] did not specifically refer to the typical sequel of a CS; nonetheless, since the main parameters examined in those studies (myoglobinuria, nerve paralysis) were strongly related to CS pathophysiology, it was decided that they should be included.

Forty-one cases were described in total, including 22 after arthroplasty of the hip [14, 16, 18, 20, 23, 25-30], 18 of the knee [1, 12-15, 17, 21-24], and 1 of the forefoot [19]. Five different anatomical areas were involved including gluteal $[14,16,17,26,28-30]$, thigh $[1,20,23,24,27]$, calf $[1,12,13,15,21,22,25]$, forefoot [19], and forearm compartments [18]. Twenty-five patients were males and 15 females with an overall average of 54.8 years of age (range 37-75). Degenerative arthritis was the reason for arthroplasty in the majority of cases (23/40) followed by loosening of implants (8/40), Avascular Necrosis (AVN) (3/40) and other reasons.

Of the 22 patients that underwent total hip arthroplasty (THA), 16 (72.7\%) developed CS of the gluteal compartment (buttocks), $4(18.2 \%)$ of the thigh, and one $(4.5 \%)$ of the calf. From the 18 patients that underwent a total knee arthroplasty (TKA), $4(22.2 \%)$ developed a CS in the gluteal area, $3(16.6 \&)$ in the thigh, and $11(61.1 \%)$ in the calf compartments (Table 3). A forefoot CS was recorded after revision forefoot arthroplasty, as well as an arm CS after a revision THA.

The mean operating time was $196 \min (65-420)$, and the mean time from surgery until the CS diagnosis was 26 hours $(0-72)[1,13-18,21,27,29]$. The mean time to fasciotomy was 53.2 hours $(8-144)$ [1, 12-21, 23, 25, 26, $28,30]$. In the cases that epidural analgesia was used, the mean time to its discontinuation was 30.8 hours $(12-48)$ [1, $12,14-17,21]$. Neurovascular parameters were recorded in some of the studies identifying absence of peripheral pulses just $13 \%(4 / 31)$ of all cases $[1,13,15-21,23,24,28-30]$, and nerve deficit (sensory or motor) in $68.42 \%(26 / 38)$ [1, 13-20, 23-30]. Intracompartmental pressure (ICP) measurement data were recorded in 6 of the 20 studies ( 8 cases) $[1,20,21$, $23-25,28$ ] with readings ranging from 32 to $110 \mathrm{~mm} \mathrm{Hg}$. In two other reports $[15,18]$ ICP measurement was not conducted, as it was considered unnecessary, due to the presence of clear clinical signs. In all cases where the ICP was recorded, it was found to be elevated leading to subsequent fasciotomies. Full recovery without any secondary sequel of CS was recorded in $52.63 \%$ of the cases $(20 / 38)[1,14,18-20,23,24,26,28-30]$. In contrast, $36.8 \%$ $(14 / 38)$ developed motor deficits (most usually a drop foot) $[1,13,14,16,17,21,23,27,28]$, and $26.3 \%(10 / 38)$ of the patients a sensory deficit (numbness, paresthesias) [12, 13, $17,21,23,27,28,30]$. One patient eventually [1] had a below the knee amputation because of irreversible equinus deformity and foot drop (Table 2).

The aetiology of CS was not clearly defined in all cases and mostly retrospective speculative assumptions were recorded. Prolonged positioning of the patients either intraoperatively (lateral decubitus) $[18,25,28,29]$, or during the nursing phase (supine, semirecumbent) [14, 17] was considered exclusively responsible for the gluteal CS after THA or TKA. Bleeding predisposition either related to long term drug administration (warfarin [20, 23]/aspirin [14]), or routine thromboprofylaxis (low molecular weight heparin subcutaneously $[13,20,23,25]$, oral warfarin [24], or intravenous heparin [30]) was mainly related to CS of the thigh $[20,23,24,27]$, or buttock $[14,30]$. Out of 26 cases that recorded the type of post-operative analgesia, continuous epidural infusion was used in 17, and was considered as responsible for a delayed CS diagnosis in 5 of them $[13-16,21]$. A tourniquet was used in 16 cases (at a level not higher than $350 \mathrm{mmHg}$ ) of TKA, or unicompartmental knee arthroplasty (UKA) and in 1 case of forefoot arthroplasty. Its use was considered responsible for the consequent CS for only 1 patient [15].

Obesity for 9 patients [14, 29], vascular thrombosis compression or rupture for 9 patients $[1,20,21,25,26,30]$, extensive soft tissue dissection for 6 patients $[1,20,23,26]$, scarring due to previous operations for 4 patients $[1,19]$, vigorous early physiotherapy for 2 patients [17, 24] and intra-operative use of calf stimulators for 1 patient [25] were reported as causative factors for CS after elective lower limb joint arthroplasty.

\section{DISCUSSION}

The pathophysiology of compartment syndrome is characterised by a unique type of ischemia that affects a group of muscles enclosed within a relatively nonexpandable fascial sheath and bony structures [31]. Its early signs include severe pain disproportionate to the injury, exacerbated by passive stretching of the involved muscles. Paresthesias may occur at an early or later stage. Swelling, coldness, and paralysis follow if surgical intervention is postponed [1]. Pulseness may or may not occur, thus the 
Table 2. Description of the Parameters of the Reviewed Studies

\begin{tabular}{|c|c|c|c|c|c|c|c|c|c|c|c|c|c|c|c|c|c|c|c|c|c|}
\hline$\frac{\pi}{ส}$ & 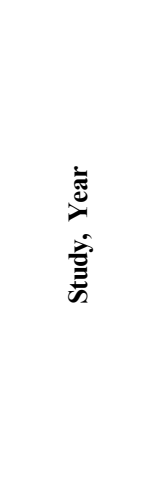 & 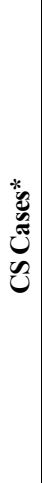 & 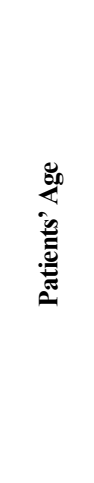 & 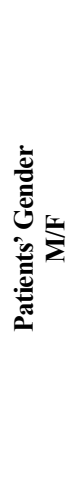 & 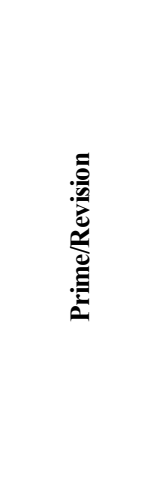 & 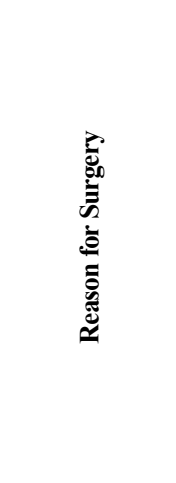 & 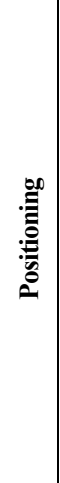 & 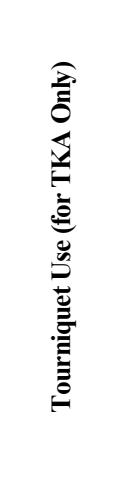 & 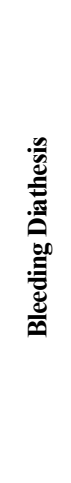 & 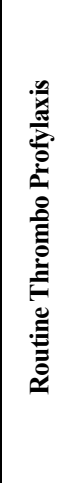 & 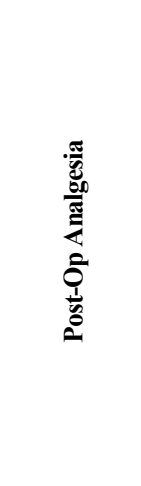 & 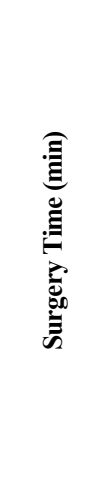 & 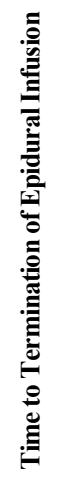 & 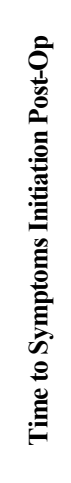 & 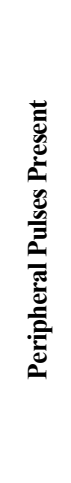 & 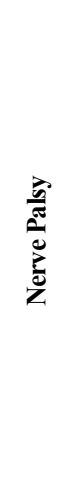 & 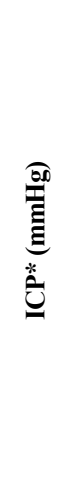 & 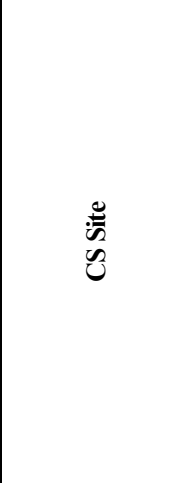 & 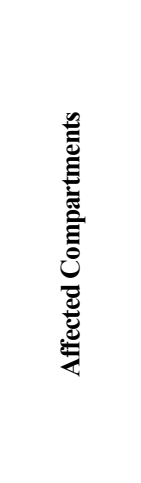 & 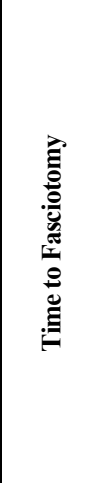 & 节 \\
\hline 1 & $\begin{array}{l}\text { Fleming, } \\
1979[30]\end{array}$ & 1 & 62 & $1 / 0$ & 1 pr. THA & DA $\times 1$ & $\mathrm{n} / \mathrm{a}$ & - & $\begin{array}{l}\text { YES } \\
\mathrm{x} 1\end{array}$ & $\begin{array}{l}\text { YES } \\
\mathrm{x} 1\end{array}$ & $\mathrm{n} / \mathrm{a}$ & $\mathrm{n} / \mathrm{a}$ & $\mathrm{n} / \mathrm{a}$ & $36 \mathrm{~h}$ & YES & YES & $\mathrm{n} / \mathrm{a}$ & $\begin{array}{l}\text { Ipsilateral } \\
\text { buttock } \\
\text { x } 1\end{array}$ & $\mathrm{n} / \mathrm{a}$ & $39 \mathrm{~h}$ & $\begin{array}{c}\text { Full recovery } \\
\quad \times 1\end{array}$ \\
\hline 2 & $\begin{array}{l}\text { Smith, } \\
1989 \text { [29] }\end{array}$ & 5 & $\begin{array}{c}48.6 \\
\text { (mean) }\end{array}$ & $5 / 0$ & $\begin{array}{l}2 \text { pr. THA } \\
3 \text { rev. THA }\end{array}$ & $\begin{array}{c}\text { DA x } 2 \\
\text { AVN x } 3\end{array}$ & $\begin{array}{l}\mathrm{LD} \\
\mathrm{x} 5\end{array}$ & - & $\mathrm{n} / \mathrm{a}$ & $\mathrm{n} / \mathrm{a}$ & $\mathrm{n} / \mathrm{a}$ & $\begin{array}{c}387.5 \\
\text { (mean) }\end{array}$ & $\mathrm{n} / \mathrm{a}$ & $\mathrm{n} / \mathrm{a}$ & $\begin{array}{l}\text { YES } \\
\times 5\end{array}$ & $\begin{array}{l}\text { YES } \\
\mathrm{x} 5\end{array}$ & $\mathrm{n} / \mathrm{a}$ & $\begin{array}{l}\text { Contralateral } \\
\text { buttock } \\
\text { x } 5\end{array}$ & $\mathrm{n} / \mathrm{a}$ & \begin{tabular}{|c|} 
not \\
per- \\
formed
\end{tabular} & $\begin{array}{l}\text { Full recovery } \\
\quad \times 5\end{array}$ \\
\hline 3 & $\begin{array}{c}\text { Lachiewicz, } \\
1991 \text { [28] }\end{array}$ & 6 & $\begin{array}{c}53.83 \\
\text { (mean) }\end{array}$ & $4 / 2$ & $\begin{array}{l}1 \mathrm{pr} \text {. THA } \\
5 \mathrm{rev} \text {. THA }\end{array}$ & $\begin{array}{l}\text { Loosening x } 4 \\
\text { Infection x } 1 \\
\text { Post traumatic } \\
\text { arthritis x } 1\end{array}$ & $\begin{array}{l}\mathrm{LD} \\
\times 6\end{array}$ & - & $\mathrm{n} / \mathrm{a}$ & $\mathrm{n} / \mathrm{a}$ & $\mathrm{n} / \mathrm{a}$ & $\mathrm{n} / \mathrm{a}$ & $\mathrm{n} / \mathrm{a}$ & $24 \mathrm{~h}$ & $\begin{array}{l}\text { YES } \\
\text { x } 6\end{array}$ & $\begin{array}{l}\text { YES } \\
\mathrm{x} 4 \\
\mathrm{NO} \\
\mathrm{x} 2\end{array}$ & $\begin{array}{l}50 \\
\mathrm{n} / \mathrm{a}\end{array}$ & $\begin{array}{c}\text { Contralateral } \\
\text { buttock } \\
\text { x } 6\end{array}$ & $\mathrm{n} / \mathrm{a}$ & $\begin{array}{l}24 \mathrm{~h} \\
\mathrm{CT}\end{array}$ & $\begin{array}{c}\text { Sensory } \\
\text { deficit } \\
\text { x } 2 \\
\text { Full recovery } \\
\text { x } 4\end{array}$ \\
\hline 4 & $\begin{array}{l}\text { Nicholl, } \\
1996 \text { [25] }\end{array}$ & 1 & 69 & $1 / 0$ & $1 \mathrm{rev}$. THA & $\begin{array}{c}\text { Loosening } \\
\quad \times 1\end{array}$ & $\begin{array}{l}\mathrm{LD} \\
\mathrm{x} 1\end{array}$ & - & $\begin{array}{l}\mathrm{NO} \\
\mathrm{x} 1\end{array}$ & $\begin{array}{l}\text { YES } \\
\mathrm{x} 1\end{array}$ & $\begin{array}{c}\text { Continuous } \\
\text { Epidural }\end{array}$ & $\mathrm{n} / \mathrm{a}$ & $\mathrm{n} / \mathrm{a}$ & $24 \mathrm{~h}$ & $\mathrm{n} / \mathrm{a}$ & YES & 32 & $\begin{array}{c}\text { Ipsilateral Tibial } \\
\text { calf } \\
\text { x } 1\end{array}$ & $\begin{array}{c}\text { Anterior } \\
\mathrm{x} 1\end{array}$ & $72 \mathrm{~h}$ & $\begin{array}{c}\text { Motor deficit } \\
\quad \times 1\end{array}$ \\
\hline 5 & $\begin{array}{c}\text { Pai, } \\
1996[26]\end{array}$ & 1 & 68 & $0 / 1$ & 1 pr. THA & DA $\times 1$ & $\mathrm{n} / \mathrm{a}$ & - & $\begin{array}{l}\mathrm{NO} \\
\mathrm{x} 1\end{array}$ & $\mathrm{n} / \mathrm{a}$ & $\begin{array}{c}\text { IM } \\
\text { Morphine }\end{array}$ & $\mathrm{n} / \mathrm{a}$ & $\mathrm{n} / \mathrm{a}$ & $12 \mathrm{~h}$ & $\mathrm{n} / \mathrm{a}$ & YES & $\mathrm{n} / \mathrm{a}$ & $\begin{array}{l}\text { Ipsilateral } \\
\text { buttock } \\
\text { x } 1\end{array}$ & $\begin{array}{l}\text { Gluteus } \\
\text { Maximus } \\
\text { x } 1\end{array}$ & $20 \mathrm{~h}$ & $\begin{array}{l}\text { Full recovery } \\
\quad \times 1\end{array}$ \\
\hline 6 & $\begin{array}{c}\text { Tani, } \\
1996[27]\end{array}$ & 1 & 73 & $0 / 1$ & $1 \mathrm{rev}$. THA & $\begin{array}{l}\text { Loosening } \\
\text { x } 1\end{array}$ & $\mathrm{n} / \mathrm{a}$ & - & $\begin{array}{l}\text { YES } \\
\times 1\end{array}$ & $\mathrm{n} / \mathrm{a}$ & $\mathrm{n} / \mathrm{a}$ & 420 & $\mathrm{n} / \mathrm{a}$ & $0 \mathrm{~h}$ & $\mathrm{n} / \mathrm{a}$ & YES & $\mathrm{n} / \mathrm{a}$ & $\begin{array}{c}\text { Ipsilateral Thigh } \\
\mathrm{x} 1\end{array}$ & $\begin{array}{c}\text { Anterior \& } \\
\text { Posterior } \\
\text { x } 1\end{array}$ & $\mathrm{CT}$ & $\begin{array}{l}\text { Sensory \& } \\
\text { Motor deficit } \\
\quad \text { x } 1\end{array}$ \\
\hline 7 & $\begin{array}{l}\text { Smith, } \\
1997[24]\end{array}$ & 1 & 66 & $1 / 0$ & 1 pr. TKA & DA $\times 1$ & $\begin{array}{c}\mathrm{Sx} \\
1\end{array}$ & $\begin{array}{l}\text { YES (75 } \\
\text { min) }\end{array}$ & $\begin{array}{l}\mathrm{NO} \\
\mathrm{x} 1\end{array}$ & $\begin{array}{l}\text { YES } \\
\mathrm{x} 1\end{array}$ & $\begin{array}{c}\text { IM } \\
\text { Morphine }\end{array}$ & $\mathrm{n} / \mathrm{a}$ & $\mathrm{n} / \mathrm{a}$ & $24 \mathrm{~h}$ & YES & NO & 35 & $\begin{array}{c}\text { Ipsilateral Thigh } \\
\text { x } 1\end{array}$ & $\begin{array}{l}\text { Anterior } \\
\mathrm{x} 1\end{array}$ & $\mathrm{CT}$ & $\begin{array}{c}\text { Full recovery } \\
\times 1\end{array}$ \\
\hline 8 & $\begin{array}{l}\text { Nadeem, } \\
1998 \text { [23] }\end{array}$ & 3 & $\begin{array}{c}69 \\
\text { (mean) }\end{array}$ & $2 / 1$ & $\begin{array}{l}2 \text { rev. THA } 1 \\
\text { pr. TKA }\end{array}$ & $\begin{array}{l}\text { Reconversion } \\
\text { from } \\
\text { Girdlestone's x } \\
1 \\
\text { Loosening x } 1 \\
\text { DA x } 1\end{array}$ & $\mathrm{n} / \mathrm{a}$ & $\mathrm{n} / \mathrm{a}$ & $\begin{array}{l}\text { YES } \\
\mathrm{x} 1 \\
\text { NO } \\
\mathrm{x} 2\end{array}$ & $\begin{array}{l}\text { YES } \\
\mathrm{x} 1\end{array}$ & Opioids pos & $\mathrm{n} / \mathrm{a}$ & $\mathrm{n} / \mathrm{a}$ & $72 \mathrm{~h}$ & $\begin{array}{l}\text { YES } \\
\times 3\end{array}$ & $\begin{array}{l}\text { YES } \\
\mathrm{x} 2 \\
\mathrm{NO} \\
\mathrm{x} 1\end{array}$ & $\begin{array}{l}105 \\
110 \\
\mathrm{n} / \mathrm{a}\end{array}$ & $\begin{array}{c}\text { Ipsilateral Thigh } \\
\times 3\end{array}$ & $\begin{array}{l}\text { Anterior } \\
\text { x } 1 \\
\text { Anterior \& } \\
\text { Posterior } \\
\text { x } 2\end{array}$ & $\begin{array}{l}96 \mathrm{~h}, \\
144 \mathrm{~h}, \\
72 \mathrm{~h}\end{array}$ & $\begin{array}{c}\text { Sensory \& } \\
\text { Motor deficit } \\
\text { x } 1 \\
\text { Motor deficit } \\
\text { x } 1 \\
\text { Full recovery } \\
\text { x } 1\end{array}$ \\
\hline
\end{tabular}


(Table 2) contd.....

\begin{tabular}{|c|c|c|c|c|c|c|c|c|c|c|c|c|c|c|c|c|c|c|c|c|c|}
\hline สี & 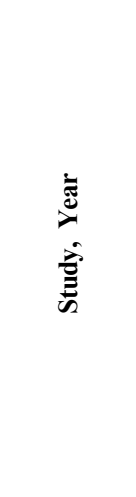 & 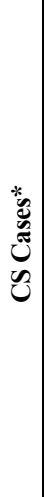 & 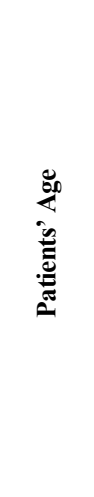 & 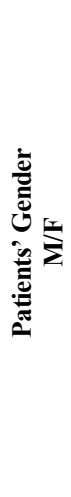 & 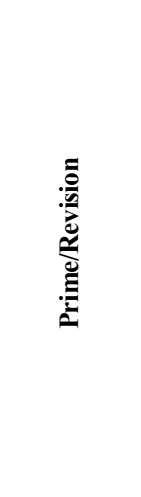 & 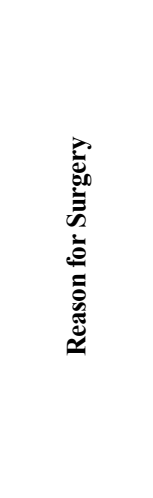 & 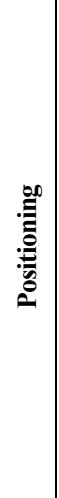 & 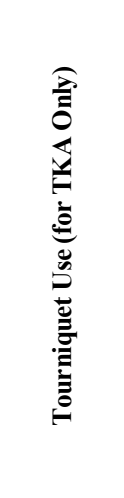 & 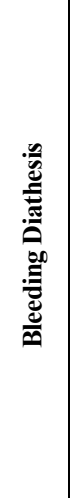 & 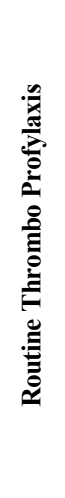 & 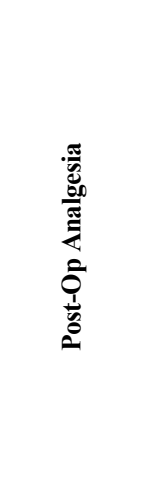 & 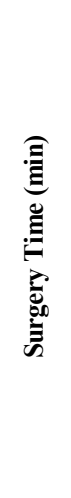 & 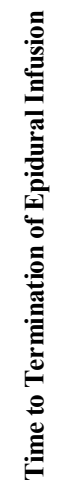 & 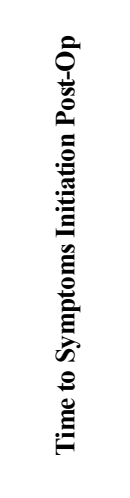 & 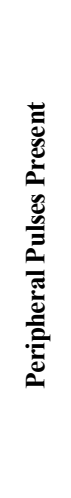 & 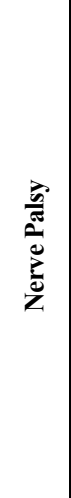 & 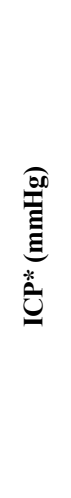 & $\begin{array}{l}\text { : } \\
\text { ¿ } \\
0\end{array}$ & 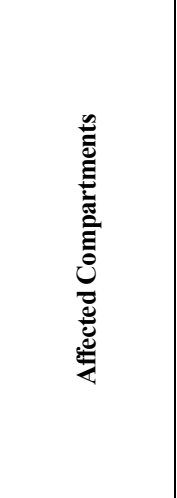 & 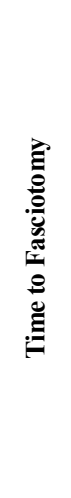 & $\begin{array}{l}\stackrel{\Xi}{0} \\
\stackrel{\Xi}{0}\end{array}$ \\
\hline 9 & $\begin{array}{c}\text { Burki, } \\
1999 \text { [22] }\end{array}$ & 1 & 75 & $0 / 1$ & 1 pr. TKA & DA $x 1$ & $\mathrm{n} / \mathrm{a}$ & $\mathrm{n} / \mathrm{a}$ & $\mathrm{n} / \mathrm{a}$ & $\mathrm{n} / \mathrm{a}$ & $\mathrm{n} / \mathrm{a}$ & $\mathrm{n} / \mathrm{a}$ & $\mathrm{n} / \mathrm{a}$ & $\mathrm{n} / \mathrm{a}$ & $\mathrm{n} / \mathrm{a}$ & $\mathrm{n} / \mathrm{a}$ & $\mathrm{n} / \mathrm{a}$ & $\begin{array}{c}\text { Ipsilateral } \\
\text { Tibial calf } \\
\text { x } 1 \\
\end{array}$ & $\begin{array}{c}\text { Anterior } \\
\mathrm{x} 1\end{array}$ & $\mathrm{n} / \mathrm{a}$ & \begin{tabular}{|c|} 
Full \\
recovery \\
x 1 \\
\end{tabular} \\
\hline 10 & $\begin{array}{c}\text { Mai, } \\
2000[20]\end{array}$ & 1 & 60 & $1 / 0$ & 1 pr. THA & DA x 1 & $\mathrm{n} / \mathrm{a}$ & - & $\begin{array}{c}\text { YES } \\
\times 1\end{array}$ & $\begin{array}{l}\text { YES } \\
\times 1\end{array}$ & $\begin{array}{c}\text { IM } \\
\text { Morphine }\end{array}$ & $\mathrm{n} / \mathrm{a}$ & $\mathrm{n} / \mathrm{a}$ & $24 \mathrm{~h}$ & YES & NO & 45.6 & $\begin{array}{c}\text { Ipsilateral } \\
\text { Thigh } \\
\text { x } 1\end{array}$ & $\begin{array}{c}\text { Anterior } \\
\mathrm{x} 1\end{array}$ & $24 \mathrm{~h}$ & $\begin{array}{l}\text { Full } \\
\text { recovery } \\
\mathrm{x} 1\end{array}$ \\
\hline 11 & $\begin{array}{l}\text { Marsh, } \\
2000[18]\end{array}$ & 1 & 68 & $1 / 0$ & $1 \mathrm{rev}$. THA & $\begin{array}{l}\text { Loosening } \\
\quad \text { X } 1\end{array}$ & $\begin{array}{l}\text { LD } \\
\times 1\end{array}$ & - & $\begin{array}{l}\text { NO } \\
\mathrm{x} 1\end{array}$ & $\begin{array}{l}\text { NO } \\
\mathrm{x} 1\end{array}$ & $\mathrm{n} / \mathrm{a}$ & 360 & $\mathrm{n} / \mathrm{a}$ & $0 \mathrm{~h}$ & YES & YES & $\mathrm{n} / \mathrm{a}$ & $\begin{array}{c}\text { Contralateral } \\
\text { Arm } \\
\text { x } 1\end{array}$ & $\begin{array}{l}\text { Anterior \& } \\
\text { Posterior } \\
\text { x } 1\end{array}$ & $8 \mathrm{~h}$ & $\begin{array}{l}\text { Full } \\
\text { recovery } \\
\text { x } 1\end{array}$ \\
\hline 12 & $\begin{array}{l}\text { Noorpuri, } \\
2000 \text { [19] }\end{array}$ & 1 & 37 & $0 / 1$ & $\begin{array}{c}1 \mathrm{rev.} \\
\text { forefoot } \\
\text { arthroplasty }\end{array}$ & $\begin{array}{c}\text { Rheumatoid } \\
\text { arthritis x } 1\end{array}$ & $\mathrm{n} / \mathrm{a}$ & YES & $\mathrm{n} / \mathrm{a}$ & $\mathrm{n} / \mathrm{a}$ & Opioids pos & $\mathrm{n} / \mathrm{a}$ & $\mathrm{n} / \mathrm{a}$ & $12 \mathrm{~h}$ & YES & YES & $\mathrm{n} / \mathrm{a}$ & $\begin{array}{c}\text { Ipsilateral } \\
\text { Forefoot } \\
\text { x } 1\end{array}$ & $\begin{array}{l}\text { Medial, } \\
\text { Interosseous, } \\
\text { Central, } \\
\text { Lateral } \\
\text { x 1 }\end{array}$ & $12 \mathrm{~h}$ & $\begin{array}{l}\text { Full } \\
\text { recovery } \\
\text { x } 1\end{array}$ \\
\hline 13 & $\begin{array}{c}\text { Tang, } \\
2000[21]\end{array}$ & 1 & 62 & $0 / 1$ & 1 pr. TKA & DA x 1 & $\mathrm{n} / \mathrm{a}$ & $\begin{array}{c}\text { YES } \\
\text { (300mm } \\
\text { Hg for } \\
100 \mathrm{~min})\end{array}$ & $\begin{array}{l}\text { NO } \\
\times 1\end{array}$ & $\mathrm{n} / \mathrm{a}$ & $\begin{array}{c}\text { Continuous } \\
\text { Epidural }\end{array}$ & 150 & $48 \mathrm{~h}$ & $\begin{array}{c}\text { no } \\
\text { symptoms } \\
\text { recorded }\end{array}$ & YES & $\mathrm{n} / \mathrm{a}$ & 65 & $\begin{array}{l}\text { Ipsilateral } \\
\text { Tibial calf } \\
\quad \text { x } 1\end{array}$ & $\begin{array}{c}\text { all } 4 \text { Tibial } \\
\text { compartments } \\
\text { x } 1\end{array}$ & $48 \mathrm{~h}$ & $\begin{array}{c}\text { Sensory \& } \\
\text { Motor } \\
\text { deficit } \\
\text { x } 1\end{array}$ \\
\hline 14 & $\begin{array}{l}\text { Pacheco, } \\
2001 \text { [17] }\end{array}$ & 2 & $\begin{array}{c}59 \\
\text { (mean) }\end{array}$ & $2 / 0$ & 2 pr. TKA & DA $x 2$ & \begin{tabular}{c|}
$\mathrm{S} x$ \\
1 \\
$\mathrm{SR}$ \\
$\mathrm{x} 1$
\end{tabular} & $\begin{array}{l}\text { YES }(112 \\
\& 110 \\
\min )\end{array}$ & $\begin{array}{l}\text { NO } \\
\times 2\end{array}$ & $\mathrm{n} / \mathrm{a}$ & $\begin{array}{c}\text { Continuous } \\
\text { Epidural }\end{array}$ & $\begin{array}{l}135 \\
145\end{array}$ & $\begin{array}{l}22 \mathrm{~h}, \\
43 \mathrm{~h}\end{array}$ & $\begin{array}{l}29 \mathrm{~h} \\
47.5 \mathrm{~h}\end{array}$ & $\begin{array}{l}\text { YES } \\
\mathrm{x} 2\end{array}$ & $\begin{array}{c}\text { YES } \\
\mathrm{x} 1 \\
\mathrm{NO} \\
\mathrm{x} 1\end{array}$ & $\mathrm{n} / \mathrm{a}$ & $\begin{array}{l}\text { Bilateral } \\
\text { buttocks } \\
\text { x 1 } \\
\text { Ipsilateral } \\
\text { buttock } \\
\text { x } 1\end{array}$ & $\begin{array}{l}\text { Gluteus } \\
\text { maximus } \\
\text { x } 2\end{array}$ & $44 \mathrm{~h}$ & \begin{tabular}{|c} 
Sensory \\
deficit \\
x 1 \\
Sensory \& \\
Motor \\
deficit \\
x 1
\end{tabular} \\
\hline 15 & $\begin{array}{c}\text { Somayaji, } \\
2005 \text { [16] }\end{array}$ & 1 & 39 & $1 / 0$ & 1 pr. THA & $\begin{array}{l}\text { Congenital } \\
\text { dysplasia x } 1\end{array}$ & $\begin{array}{l}\text { LD } \\
\times 1\end{array}$ & - & $\begin{array}{l}\text { NO } \\
\times 1\end{array}$ & $\mathrm{n} / \mathrm{a}$ & $\begin{array}{c}\text { Continuous } \\
\text { Epidural }\end{array}$ & 120 & $36 \mathrm{~h}$ & $36 \mathrm{~h}$ & YES & YES & $\mathrm{n} / \mathrm{a}$ & $\begin{array}{c}\text { Bilateral } \\
\text { buttocks } \\
\text { x } 1\end{array}$ & $\begin{array}{l}\text { bilateral } \\
\text { Gluteus } \\
\text { maximus } \\
\text { x } 1\end{array}$ & $60 \mathrm{~h}$ & $\begin{array}{c}\text { Motor } \\
\text { deficit } \\
\text { x } 1\end{array}$ \\
\hline
\end{tabular}


(Table 2) contd....

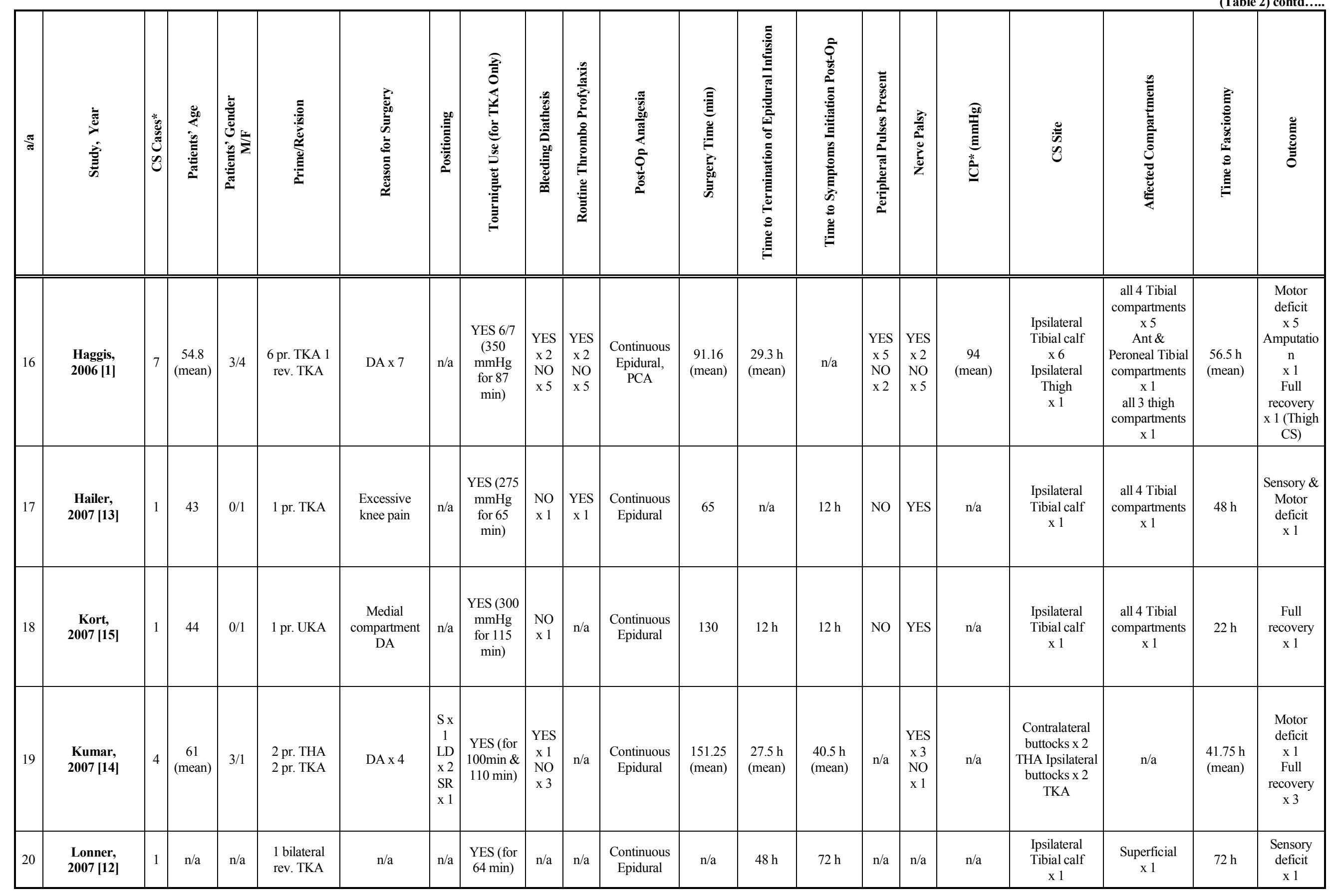


(Table 2) contd....

\begin{tabular}{|c|c|c|c|c|c|c|c|c|c|c|c|c|c|c|c|c|c|c|c|c|c|}
\hline$\frac{\pi}{\sigma}$ & 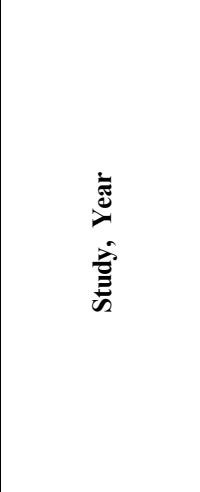 & 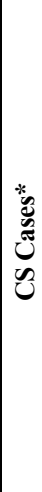 & 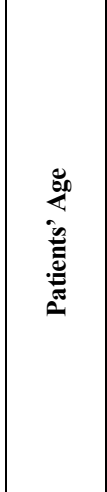 & 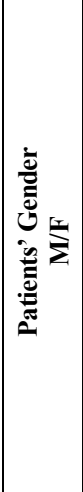 & 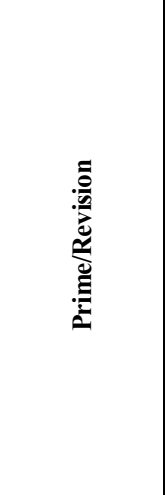 & 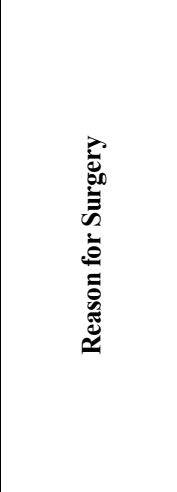 & 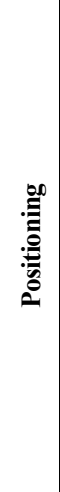 & 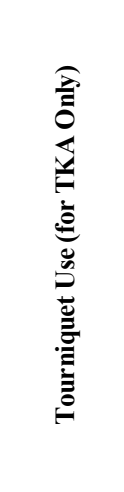 & 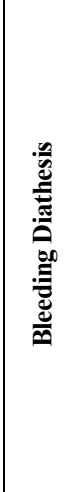 & 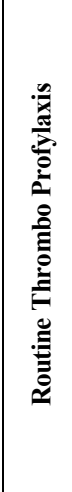 & 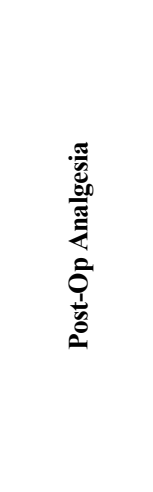 & 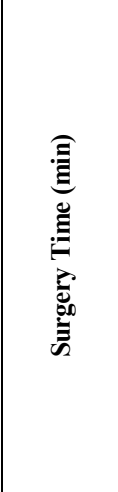 & 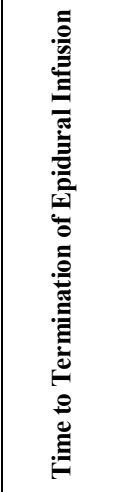 & 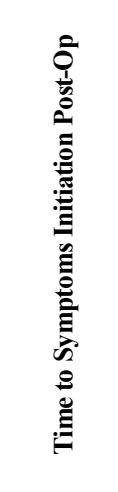 & 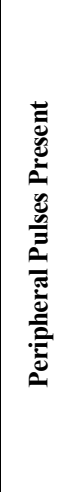 & $\begin{array}{l}\frac{n}{n} \\
\frac{0}{\pi} \\
\frac{d}{0} \\
z\end{array}$ & 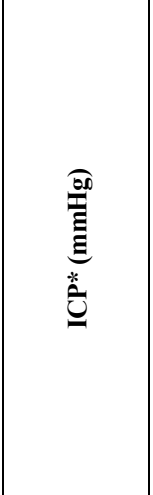 & $\begin{array}{l}\text { 离 } \\
\text { D }\end{array}$ & 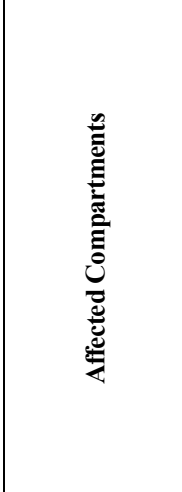 & 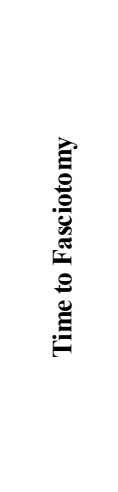 & ڤ્气 \\
\hline & $\begin{array}{c}\text { SUM/AVERAG } \\
\text { E }\end{array}$ & 41 & $\begin{array}{c}54.8 \\
(\text { mean })\end{array}$ & $25 / 15$ & $\begin{array}{c}9 \mathrm{pr} \text {. THA } \\
13 \text { rev. THA } \\
15 \text { pr. TKA } \\
2 \text { rev. TKA } \\
1 \text { pr. UKA } \\
1 \text { rev. } \\
\text { Forefoot }\end{array}$ & $\begin{array}{c}\text { DA x } 23 \\
\text { Loosening x } 8 \\
\text { AVN x } 3 \\
\text { other x } 6\end{array}$ & $\begin{array}{c}\text { Sx } \\
3 \\
\text { LD } \\
x \\
16 \\
\text { SR } \\
\text { x } 2 \\
\text { n/a } \\
x \\
21\end{array}$ & $\begin{array}{c}\text { TIME } \\
\text { Average } \\
91.5 \text { min } \\
\text { Range } \\
\text { 64-112 } \\
\text { min }\end{array}$ & \begin{tabular}{|c|} 
YES \\
x 7 \\
NO \\
x 20 \\
n/a x \\
14
\end{tabular} & $\begin{array}{c}\text { YES } \\
\text { x } 8 \\
\text { NO } \\
\text { x } 6 \\
\text { n/a x } \\
25\end{array}$ & $\begin{array}{c}\text { Continuous } \\
\text { Epidural } \\
\text { x } 18 \\
\text { IM } \\
\text { Morphine } \\
\text { x 3 } \\
\text { Opioids pos } \\
\text { x 4 } \\
\text { PCA } \\
\text { x 1 } \\
\text { n/a } \\
\text { x 15 }\end{array}$ & $\begin{array}{c}\text { Average } \\
\text { 196min } \\
\text { Range } \\
65- \\
420 \mathrm{~min}\end{array}$ & $\begin{array}{l}\text { Average } \\
30.8 \mathrm{~h} \\
\text { Range } \\
12-48 \mathrm{~h}\end{array}$ & $\begin{array}{c}\text { Average } \\
\text { 26 h } \\
\text { Range } \\
0-72 ~ h\end{array}$ & $\begin{array}{l}\text { YES } \\
\text { x } 28 \\
\text { NO } \\
\text { x } 4 \\
\text { n/a } \\
\times 9\end{array}$ & $\begin{array}{c}\text { YES } \\
\text { x 26 } \\
\text { NO } \\
\text { x 12 } \\
\text { n/a } \\
\text { x } 3\end{array}$ & \begin{tabular}{|c|} 
Average \\
78.6mmHg \\
Range \\
32-110h
\end{tabular} & $\begin{array}{c}\text { Buttocks: } \mathbf{x} \mathbf{2 0} \\
5 \text { ipsil, } 13 \text { con., } \\
2 \text { bil. } \\
\text { Thigh: } \mathbf{x} 7 \\
7 \text { ipsil, } 0 \text { con. } \\
\text { Tibial calf: } \mathbf{x} \\
\mathbf{1 2} \\
12 \text { ipsil, } 0 \text { con. } \\
\text { Forefoot: } \mathbf{1} \\
1 \text { ipsil, } 0 \text { con } \\
\text { Arm: } \mathbf{1} \\
1 \text { ipsil, } 0 \text { con. }\end{array}$ & $\begin{array}{c}\text { Buttock } \\
\text { Gluteus } \\
\text { maximus x } 6 \\
\text { Thigh } \\
\text { Anterior x } 7 \\
\text { Posterior x } 4 \\
\text { Medial x } 1 \\
\text { Tibial calf } \\
\text { Anterior x } 13 \\
\text { Peroneal x } 11 \\
\text { Superficial x } \\
11 \\
\text { Deep Posterior } \\
\text { x } 10\end{array}$ & $\begin{array}{l}\text { Average } \\
53.2 \text { h } \\
\text { Range } \\
\text { 8-144 h }\end{array}$ & $\begin{array}{c}\text { Full } \\
\text { Recovery } \\
\text { x 22 } \\
\text { Sensory } \\
\text { deficit x 4 } \\
\text { Motor } \\
\text { deficit x 9 } \\
\text { Sensory } \\
\text { \& Motor } \\
\text { deficit x } 5 \\
\text { Amputati } \\
\text { on x 1 }\end{array}$ \\
\hline
\end{tabular}

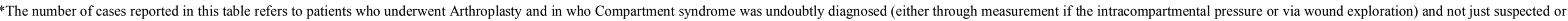
hypothesized.

\section{Abbreviations:}

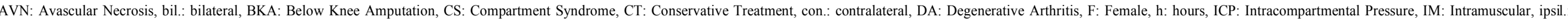

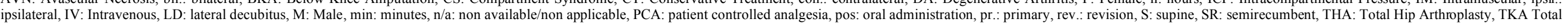
Knee Arthroplasty, UKA: Unilateral Knee Arthroplasty

* The recorded value of ICP refers to the highest value recorded among the compartments that were measured 
Table 3. Number of Cases in Terms of Localisation of Arthroplasty, Localisation of Compartment Syndrome (CS) \& Potential Reasons

\begin{tabular}{|c|c|c|c|c|}
\hline & Arthroplasty Joint & Gluteal CS & Thigh CS & Calf CS \\
\hline \multirow{2}{*}{ Total no of cases } & Hip & 17 & 4 & 1 \\
\hline & Knee & 4 & 3 & 11 \\
\hline \multirow{2}{*}{ Positioning } & Hip & 14 & - & - \\
\hline & Knee & 2 & - & - \\
\hline \multirow{2}{*}{ Routine thromboprofylaxis } & Hip & 1 & 4 & 1 \\
\hline & Knee & - & 2 & 2 \\
\hline \multirow{2}{*}{ Bleeding predisposition } & Hip & 1 & 3 & \\
\hline & Knee & 1 & 1 & 1 \\
\hline \multirow{2}{*}{ Aggressive Physiotherapy } & Hip & - & - & - \\
\hline & Knee & 1 & 1 & - \\
\hline \multirow{2}{*}{ Extensive soft tissue dissection } & Hip & 7 & 2 & \\
\hline & Knee & - & - & 3 \\
\hline \multirow{2}{*}{ Previous operations on site } & Hip & 8 & - & - \\
\hline & Knee & - & - & - \\
\hline \multirow{2}{*}{ Continuous Epidural anaesthesia } & Hip & 3 & - & 1 \\
\hline & Knee & 4 & - & 9 \\
\hline
\end{tabular}

presence of a peripheral pulse does not rule out the presence of CS [32]. Clinical symptoms are usually adequate to make the diagnosis. In doubtful cases, or in unconscious patients, intra-compartmental pressure measurements are helpful to set the diagnosis and to proceed with the subsequent urgent fasciotomies. Missed diagnosis and late decompression is associated with significant morbidity due to irreversible ischemic necrosis of the muscles and nerves within the compartment [33]. In neglected cases severe systemic complications may follow (myoglobinuria and acute renal failure), or even death [28].

One of the main drawbacks is that the timing of the onset of CS cannot always be precisely defined. Moreover, when CS occurs after "low risk" operations, such as joint arthroplasty, the chance of remaining undiagnosed the first valuable hours is high. This delay, may lead to dramatic consequences secondary to irreversible ischemia of the nerves and muscle tissue [34].

Our study aimed to identify and comprehensively present the existing evidence on CS aetiology related to elective joint arthroplasty of the lower limb. Despite the small number of cases which were retrieved from the published series in PubMed, useful suggestions related to CS prevention and management could be made. Firstly, it is of paramount importance that all the staff (medical and paramedical) involved in the perioperative care of lower limb arthroplasty patients is aware of the clinical presentation and importance of early diagnosis and management of compartment syndrome. Secondly, specific precautionary measures need to be applied as routine practice: i. $\quad$ Positioning: It was evident from the reviewed studies that the lateral decubitus positioning during THA surgery was highly correlated with gluteal CS. This was mainly attributed to the incorrect placement of the posterior pad causing compression on the gluteal muscles [28], or to the firm placement of the anterior clamp that pushes the contralateral thigh and occlude the femoral neurovascular bundle causing sensory and motor deficits [29]. It is characteristic that in almost all of the THA - gluteal CS cases [14, 16, 28, 29] the syndrome was developed in the contralateral buttock incriminating positioning as the causative factor, and indicating the need for careful placement of the posterior and anterior clamps, in order to avoid compression of the gluteal muscles or of the femoral triangle. Adequate padding between the clamps and the body of the patient should be applied. There were also 2 studies $[14,17]$ in which post TKA gluteal CS was attributed to postoperative nursing in a semirecumbent position. The clear message from these 2 studies was that vigilant nursing care for regular change of posture and for recognition of motor blockade is mandatory especially for obese patients under epidural analgesia. Considering CS related to patient's positioning, surgeons should always keep in mind the higher risk due to the Trendelenburg inclination. This is usually associated with concomitant lithotomy position of the legs [35] which is not used in lower limb joint arthroplasty. Nonetheless in the setting of hypotensive anaesthesia, which is by itself a predisposing factor for diminished blood perfusion, the addition of prolonged Trendelenburg inclination may create unwanted consequences and should be avoided. 
ii. Tourniquet use: Pneumatic tourniquets maintain a relatively bloodless field during knee or forefoot arthroplasty, aid identification of vital structures, and expedite the procedure [36]. However, after prolonged use they may induce an ischemiareperfusion injury causing CS $[8,36]$. Safe limits of duration under tourniquet and levels of pressure are debatable. Worland [37] stated that the tourniquet pressure should be limited to $100 \mathrm{mmHg}$ above systolic pressure, while Klenerman and Hulands [38] describe the ideal tourniquet pressure for the thigh to be double that of the systolic pressure in the arm. Moreover, they proposed the time safety limit of three hours for continuous tourniquet use. In the reviewed studies that reported CS after TKA the authors did not correlate its incidence to the tourniquet use. A possible explanation may be that the recorded tourniquet times and pressures were all well within the established safety limits.

iii. Calf compression devices: Calf compression devices have been implicated in the past for the development of compartment syndrome [39]. In one of the studies of this review [25] intraoperative use of calf stimulation devices was considered a potential reason for CS. In contrary, later evidence [40] advocated that external intermittent compression devices decrease compartment pressures by improving venal return in contrast to antithrombotic elastic stockings. Use of interchangeable calf compression devices is recommended, however their contribution in the avoidance of compartment syndrome needs to be investigated further.

iv. Physiotherapy: Post-operative physiotherapy should avoid early aggressive passive flexion of the hip or the knee. In patients post-TKA that commence early continuous passive motion, flexion to $30^{\circ}$ was found to produce dramatic pressure elevation up to 35 $\mathrm{mmHg}$ creating $\mathrm{CS}$ in the thigh or the gluteal compartment $[17,24]$. It may be preferable to restrict joint mobilisation protocols to active-assisted passive or simple active flexion-extension exercises for the first 2 days post-operatively. More aggressive apparatus assisted physiotherapy could follow since the patient would have indiscriminately overcome the first stage of mobilisation.

v. Post-op analgesia: Increasing pain despite analgesic medication is the main CS symptom and should always alert clinicians. Deep sensory and motor epidural blockade excludes pain as a constant indicator for compartment syndrome since it is inevitably no longer present. This, in combination with the low index of suspicion for CS may create a disastrous combination. It is essential, when using local epidural anaesthetics that the intensity of the block is appropriate to the anticipated intensity of the wound pain without inducing motor blockade [41]. Nonetheless, analgesia should not be misunderstood as the cause but only as being a factor delaying the diagnosis. According to a recent review [42] there was no convincing evidence that epidural analgesia or patient-controlled analgesia delays the diagnosis of compartment syndrome provided patients are adequately monitored. This matches with the findings of our review since continuous epidural analgesia was considered responsible for totally masking the symptoms in only 2 , poorly monitored, patients out of 17 that received it. Thus, it can be suggested that whatever the mode of analgesia used, a high index of clinical suspicion, ongoing assessment of patients, and compartment pressure measurement are of outmost importance for an early diagnosis [42].

vi. Anticoagulant administration: Pharmacological prophylaxis against venous thromboembolism may be associated with serious haemorrhagic risks [43]. Bleeding predisposition in patients receiving anticoagulants on a permanent base due to vascular disease should be taken into account. Those patients need meticulous monitoring throughout the perioperative period. For patients not chronically receiving anticoagulants the type and the dose of prophylaxis remains unclear with certain differences existing between American and European protocols [43]. In some of the reviewed studies the use of anticoagulants was considered as a causative factor $[23,27,30]$. Nonetheless, the correlation could not be clearly proved, and moreover other reasons, such as extensive soft tissue dissection [23] or general hypoxia of the limb [30], coexisted. Patients undergoing joint arthroplasty should receive routine anticoagulation therapy, based on the local protocols of every institute. In any case though, the coagulation profile (fibrinogen, FDP, platelets, INR) needs to be evaluated pre- and post-operatively and specialist consultation requested if necessary. As soon as a suspicion related to $\mathrm{CS}$ is raised, anticoagulation needs to be halted and its effect reversed if possible.

vii. High risk patients: In those cases where prolonged time of operation, hypotension during anaesthesia, lateral decubitus positioning, obesity, previous operations on the same joint, vascular disease are present, meticulous clinical monitoring, and if feasible, measurement of the compartment pressures, should become the standard of practice [44]. Measurement of the intracompartmental pressure (ICP) is important, but should not replace clinical judgement $[8,45]$. The diagnosis of CS is mainly clinical, however it should not be forgotten that pain may be an unreliable symptom as it is subjective and variable [42]. It may be absent in established acute compartment syndrome associated with nerve injury, or minimal in deep posterior compartment syndrome [44]. Thus, ICP measurement is useful in doubtful cases as well as in cases in which epidural analgesia may mask the symptoms. Furthermore, it is useful in identifying which of the compartments are involved. Decompression is generally recommended when the compartment pressure exceeds $30 \mathrm{mmHg}$ to 35 $\mathrm{mmHg}[8,41,44]$. The view that fasciotomy should be generally performed when tissue pressure rises over $20 \mathrm{mmHg}$ below diastolic pressure $[34,46]$ appears to be of wide acceptance lately. Deep vein thrombosis (DVT) may also be present causing thigh 
Table 4 Perioperative Assessment for Prevention of Joint Arthroplasty Related Compartment Syndrome for Conscious Patients

\section{THA - TKA}

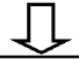

Referral to Specialised Centres

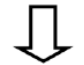

\section{Preoperative Clinical assessment}

- Check history of preexisting vascular disease \& bleeding predisposition (check coagulation profile)

- Consider obesity of the patient $(\mathrm{BMI} *>25)$

- Check for previous operations around the joint - previous scarring (revision arthroplasty or previous trauma, infection)

- Consider the psychological status of the patient (compliance to instructions for proper positioning post-op)

- Check for preexisting neurological deficit (pre-operatively existing motor or sensor deficit may mimic CS symptoms post-op)

- Individualize the method of anesthesia to be used according to the patient situation

(anesthetic pre-assessment- interdisciplinary consensus)

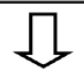

\begin{tabular}{|l|}
\hline Intraoperative assessment \\
\hline - Check correct positioning of clamps and insert adequate padding \\
- In case of lateral decubitus positioning, periodically decompress the pressure on the contralateral limbs \\
(elevation of the operated hip) \\
- Consider hypotensive episode during surgery (increased risk for CS post-op) \\
- Avoid Trendelenburg positioning of the patient at any time (increased risk for CS post-op) \\
- Avoid using electrical calf stimulators intra-operatively (increased risk of producing edema in the tibial calf) \\
- Avoid Tourniquet pressure that exceeds the double of the patient's arm pressure \\
- After the first 3 hours of surgery deflate and re-inflate Tourniquet \\
- Consider releasing the tourniquet before wound closure and provide haemostasis \\
$\square$
\end{tabular}

\section{Postoperative assessment}

- Check coagulation profile post-operatively (adjust anticoagulant administration appropriately and avoid bleeding diathesis)

- Instructions for alterations of positioning on bed (avoid treating TKA patients in semi recumbent position)

- Monitoring of the blood drainage from the surgical wound

- Avoid early aggressive physiotherapy on the hip and knee for the first 2 days

- Use external intermittent calf compression devices - Avoid constant pressure devices

* Body Mass Index: $\quad \mathrm{BMI}=\frac{\text { mass }(\mathrm{kg})}{\text { height }^{2}\left(\mathrm{~m}^{2}\right)}($ normal value $=20-25)$

Abbreviations: $\mathrm{BMI}=$ Body Mass Index, $\mathrm{CS}=$ Compartment Syndrome, $\mathrm{THA}=$ Total Hip Arthroplasty, $\mathrm{TKA}=$ Total Knee Arthroplasty

swelling and firmness on palpation. It should be excluded before proceeding to fasciotomy [47]. It has been stated that muscles tolerate well 4 hours of ischemia, by 6 hours the result is uncertain, and after 8 hours the damage is irreversible [34]. Even if these values are indicative and may vary depending on the clinical setting or the patient's profile, time is precious. The need for early fasciotomy is absolute and decompression of all the affected anatomical compartments is recommended. It should be stated however, that fasciotomies are not hazardless procedures, and there is some evidence that they may cause chronic venous insufficiency due to impairment 
Table 5. Algorithm for Management of CS After THA or TKA

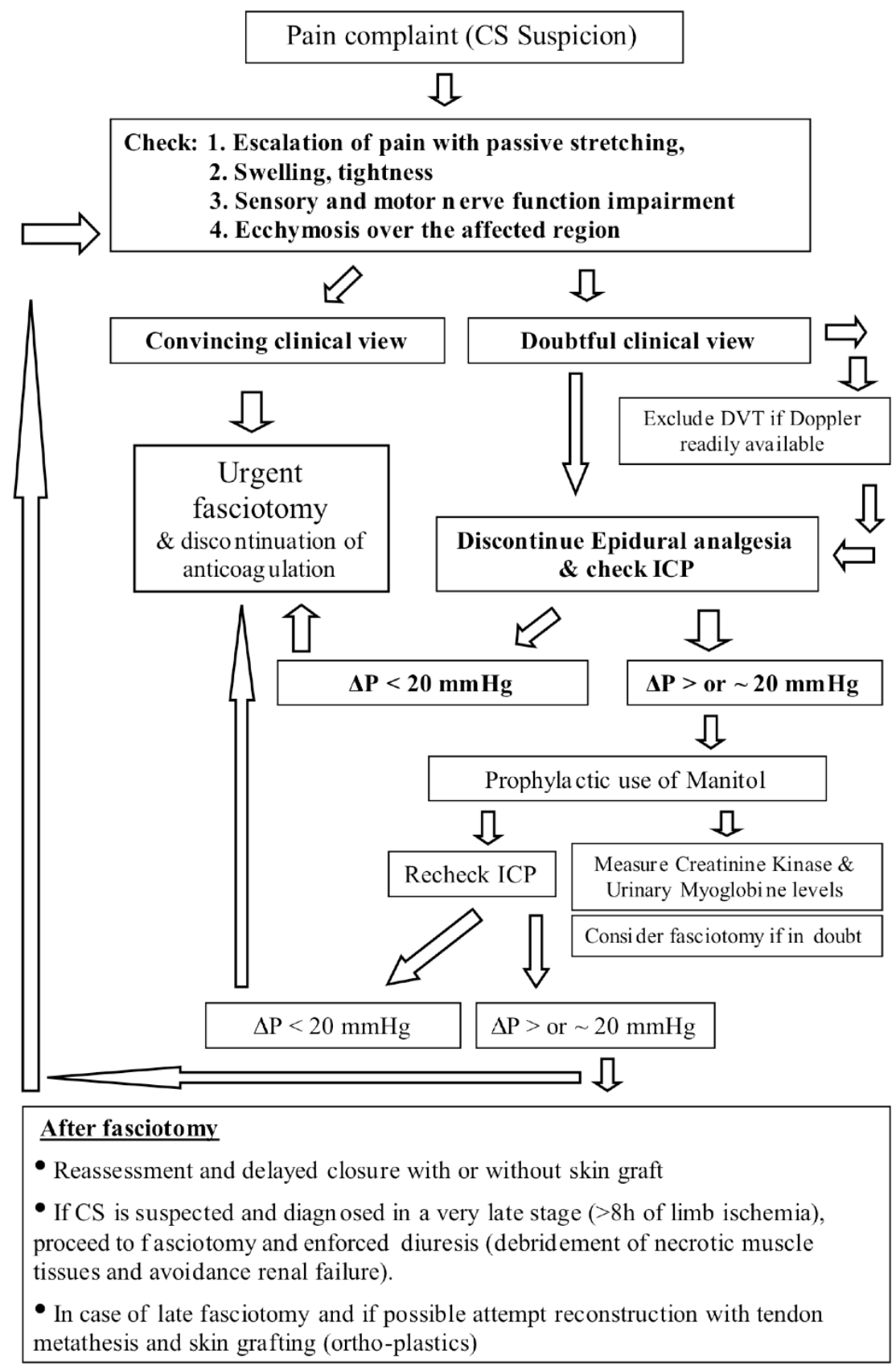

Abbreviations: $\mathrm{CS}=$ Compartment Syndrome, DVT $=$ Deep Vein Thrombosis, ICP $=$ Intracompartmental pressure, THA $=$ Total Hip Arthroplasty, TKA $=$ Total Knee Arthroplasty, $\Delta \mathrm{P}=$ Difference between the diastolic blood pressure and the Intracompartmental pressure

of the calf muscle pump, gross scars, and increase infection rates [48].

viii. Late Decompression: The role of fasciotomy in cases of compartment syndrome which has been diagnosed at a late stage (after 8 hours) is questionable. Established myoneural deficits seldom recover after late fasciotomies [49]. Furthermore, fasciotomies performed after 35 hours from injury were invariably associated with severe infection and even death [49]. Nonetheless, even if a compartment syndrome is suspected at a stage when fasciotomy may be too late, the salvation of the compartment should be attempted. The necrotic contents of the compartment need to be debrided, to avoid myoglobinuria associated complications, while early tendon metathesis combined with skin grafting may provide a reasonable functional result. 
The CK values are considered as a sensitive marker of ongoing rhabdomyolysis. CK values of over 2,000 U/1 after surgery may be considered a warning sign in ventilated and sedated patients, in whom early clinical symptoms of the compartment syndrome such as pain and paresthesias cannot be assessed [50]. The presence of myoglobinuria suggests an impending CS but the amount of myoglobinuria does not correlate with the degree of underlying tissue injury [51]. Prophylactic use of mannitol may obviate the need for decompressive fasciotomy [41], however if myoglobinuria is established enforced diuresis is recommended in order to avoid renal complications [50].

The importance of prevention and early diagnosis of the compartment syndrome is paramount for those patients at risk. Surgeons and nursing staff need to be familiar with this complication and follow strategies for its prevention (Tables 4 and 5). The rarity of CS following joint arthroplasty, and the difficulty of obtaining large prospective series limits the strength of the existing evidence. As it was previously reported [6], elective and reconstructive procedures, such as joint arthroplasty, should be performed in specialist centres where the use of specific preventive and monitoring protocols are optimal.

\section{CONFLICT OF INTEREST STATEMENT}

The authors would like to confirm that there were not any financial or personal relationships with other people or organisations that could inappropriately influence (bias) the material and the results of this manuscript.

\section{ABBREVIATIONS}

$\begin{array}{lll}\mathrm{CK}= & \text { Creatinine Kinase } \\ \mathrm{CS}= & \text { Compartment Syndrome } \\ \text { DVT } & \quad \text { Deep Vein Thrombosis } \\ \text { ICP }= & \text { Intracompartmental Pressure } \\ \text { THA }= & \text { Total Hip Arthroplasty } \\ \text { TKA }= & \text { Total Knee Arthroplasty }\end{array}$

\section{REFERENCES}

[1] Haggis P, Yates P, Blakeway C, et al. Compartment syndrome following total knee arthroplasty: a report of seven cases. J Bone Joint Surg Br 2006; 88(3): 331-4.

[2] Barei DP, Nork SE, Mills WJ, Henley MB, Benirschke SK. Complications associated with internal fixation of high-energy bicondylar tibial plateau fractures utilizing a two-incision technique. J Orthop Trauma 2004; 18(10): 649-57.

[3] Mithofer K, Lhowe DW, Vrahas MS, Altman DT, Altman GT. Clinical spectrum of acute compartment syndrome of the thigh and its relation to associated injuries. Clin Orthop Relat Res 2004; 425: 223-9.

[4] Suzuki T, Moirmura N, Kawai K, Sugiyama M. Arterial injury associated with acute compartment syndrome of the thigh following blunt trauma. Injury 2005; 36(1): 151-9.

[5] Verleisdonk EJ, Schmitz RF, van der Werken C. Long-term results of fasciotomy of the anterior compartment in patients with exercise-induced pain in the lower leg. Int J Sports Med 2004; 25(3): 224-9.

[6] Simms MS, Terry TR. Well leg compartment syndrome after pelvic and perineal surgery in the lithotomy position. Postgrad Med J 2005; 81(958): 534-6.

[7] Mullett H, Al-Abed K, Prasad CV, O'Sullivan M. Outcome of compartment syndrome following intramedullary nailing of tibial diaphyseal fractures. Injury 2001; 32(5): 411-3.
[8] Mubarak SJ, Owen CA, Hargens AR, Garetto LP, Akeson WH. Acute compartment syndromes: diagnosis and treatment with the aid of the wick catheter. J Bone Joint Surg Am 1978; 60(8): 10915 .

[9] Ebraheim NA, Hoeflinger MJ, Savolaine ER, Jackson WT. Anterior compartment syndrome of the thigh as a complication of blunt trauma in a patient on prolonged anticoagulation therapy. Clin Orthop Relat Res 1991; 263: 180-4.

[10] Kostler W, Strohm PC, Sudkamp NP. Acute compartment syndrome of the limb. Injury 2005; 36(8): 992-8.

[11] Crowninshield RD, Rosenberg AG, Sporer SM. Changing demographics of patients with total joint replacement. Clin Orthop Relat Res 2006; 443: 266-72.

[12] Lonner JH, Jasko JG, Bezwada HP, Booth RE, Jr. Morbidity of sequential bilateral revision TKA performed under a single anesthetic. Clin Orthop Relat Res 2007; 464: 151-6.

[13] Hailer NP, Adalberth G, Nilsson OS. Compartment syndrome of the calf following total knee arthroplasty--a case report of a highly unusual complication. Acta Orthop 2007; 78(2): 293-5.

[14] Kumar V, Saeed K, Panagopoulos A, Parker PJ. Gluteal compartment syndrome following joint arthroplasty under epidural anaesthesia: a report of 4 cases. J Orthop Surg (Hong Kong) 2007; 15(1): 113-7.

[15] Kort NP, van Raay JJ, van Horn JR. Compartment syndrome and popliteal vascular injury complicating unicompartmental knee arthroplasty. J Arthroplasty 2007; 22(3): 472-6.

[16] Somayaji HS, Hassan AN, Reddy K, Heatley FW. Bilateral gluteal compartment syndrome after total hip arthroplasty under epidural anesthesia. J Arthroplasty 2005; 20(8): 1081-3.

[17] Pacheco RJ, Buckley S, Oxborrow NJ, Weeber AC, Allerton K. Gluteal compartment syndrome after total knee arthroplasty with epidural postoperative analgesia. J Bone Joint Surg Br 2001; 83(5): 739-40.

[18] Marsh A, Johnstone D, Stott D. Compartment syndrome as a complication of positioning for revision hip arthroplasty--a case report. Acta Orthop Scand 2000; 71(5): 527-9.

[19] Noorpuri BS, Shahane SA, Getty CJ. Acute compartment syndrome following revisional arthroplasty of the forefoot: the dangers of ankle-block. Foot Ankle Int 2000; 21(8): 680-2.

[20] Mai DD, MacDonald SJ, Bourne RB. Compartment syndrome of the right anterior thigh after primary total hip arthroplasty. Can J Surg 2000; 43(3): 226-7.

[21] Tang WM, Chiu KY. Silent compartment syndrome complicating total knee arthroplasty: continuous epidural anesthesia masked the pain. J Arthroplasty 2000; 15(2): 241-3.

[22] Burki H, von Knoch M, Heiss C, Drobny T, Munzinger U. Lateral approach with osteotomy of the tibial tubercle in primary total knee arthroplasty. Clin Orthop Relat Res 1999; 362: 156-61.

[23] Nadeem RD, Clift BA, Martindale JP, Hadden WA, Ritchie IK. Acute compartment syndrome of the thigh after joint replacement with anticoagulation. J Bone Joint Surg Br 1998; 80(5): 866-8.

[24] Smith PN, Rampersaud R, Rorabeck CH. Incipient compartment syndrome of the thigh following total knee arthroplasty. J Arthroplasty 1997; 12(7): 835-8.

[25] Nicholl JE, Calzada S, Bonnici AV. Anterior compartment syndrome after revision hip arthroplasty. J Bone Joint Surg Br 1996; 78(5): 812-3.

[26] Pai VS. Compartment syndrome of the buttock following a total hip arthroplasty. J Arthroplasty 1996; 11(5): 609-10.

[27] Tani Y, Inoue K, Hukuda S. Acute compartment syndrome in the thigh after revision hip arthroplasty: a case report. Acta Orthop Scand 1996; 67(1): 71-2.

[28] Lachiewicz PF, Latimer HA. Rhabdomyolysis following total hip arthroplasty. J Bone Joint Surg Br 1991; 73(4): 576-9.

[29] Smith JW, Pellicci PM, Sharrock N, Mineo R, Wilson PD, Jr. Complications after total hip replacement. The contralateral limb. J Bone Joint Surg Am 1989; 71(4): 528-35.

[30] Fleming RE, Jr., Michelsen CB, Stinchfield FE. Sciatic paralysis. A complication of bleeding following hip surgery. J Bone Joint Surg Am 1979; 61(1): 37-9.

[31] Korompilias AV, Lykissas MG, Vekris MD, Beris AE, Soucacos PN. Microsurgery for lower extremity injuries. Injury 2008; 39 Suppl 3: S103-8.

[32] McQueen M. Acute compartment syndrome. Acta Chir Belg 1998; 98(4): 166-70. 
[33] Ebenezer S, Dust W. Missed acute isolated peroneal compartment syndrome. CJEM 2002; 4(5): 355-8.

[34] Whitesides TE, Heckman MM. Acute Compartment Syndrome: Update on Diagnosis and Treatment. J Am Acad Orthop Surg 1996; 4(4): 209-18.

[35] Horgan AF, Geddes S, Finlay IG. Lloyd-Davies position with Trendelenburg--a disaster waiting to happen? Dis Colon Rectum 1999; 42(7): 916-9; discussion 9-20.

[36] Wakai A, Winter DC, Street JT, Redmond PH. Pneumatic tourniquets in extremity surgery. J Am Acad Orthop Surg 2001; 9(5): 345-51.

[37] Worland RL, Arredondo J, Angles F, Lopez-Jimenez F, Jessup DE. Thigh pain following tourniquet application in simultaneous bilateral total knee replacement arthroplasty. J Arthroplasty 1997; 12(8): 848-52.

[38] Klenerman L, Biswas M, Hulands GH, Rhodes AM. Systemic and local effects of the application of a tourniquet. J Bone Joint Surg Br 1980; 62(3): 385-8

[39] Lachmann EA, Rook JL, Tunkel R, Nagler W. Complications associated with intermittent pneumatic compression. Arch Phys Med Rehabil 1992; 73(5): 482-5.

[40] Pfeffer SD, Halliwill JR, Warner MA. Effects of lithotomy position and external compression on lower leg muscle compartment pressure. Anesthesiology 2001; 95(3): 632-6.

[41] Mumtaz FH, Chew H, Gelister JS. Lower limb compartment syndrome associated with the lithotomy position: concepts and perspectives for the urologist. BJU Int 2002; 90(8): 792-9.

[42] Mar GJ, Barrington MJ, McGuirk BR. Acute compartment syndrome of the lower limb and the effect of postoperative analgesia on diagnosis. Br J Anaesth 2009; 102(1): 3-11.
[43] Hynson JM. Role of heparin in compartment syndrome. J Bone Joint Surg Am 2000; 82(5): 752-3.

[44] McQueen MM, Christie J, Court-Brown CM. Acute compartment syndrome in tibial diaphyseal fractures. J Bone Joint Surg Br 1996; 78(1): 95-8.

[45] Allen MJ, Stirling AJ, Crawshaw CV, Barnes MR. Intracompartmental pressure monitoring of leg injuries. An aid to management J Bone Joint Surg Br 1985; 67(1): 53-7.

[46] Whitesides TE, Haney TC, Morimoto K, Harada H. Tissue pressure measurements as a determinant for the need of fasciotomy. Clin Orthop Relat Res 1975; 113: 43-51.

[47] Rimoldi RL, Capen DA. Thigh compartment syndrome secondary to intertrochanteric hip fracture in a quadriplegic patient: case report. Paraplegia 1992; 30(5): 376-8.

[48] Fitzgerald AM, Gaston P, Wilson Y, Quaba A, McQueen MM. Long-term sequelae of fasciotomy wounds. Br J Plast Surg 2000; 53(8): 690-3

[49] Finkelstein JA, Hunter GA, Hu RW. Lower limb compartment syndrome: course after delayed fasciotomy. J Trauma 1996; 40(3): 342-4.

[50] Lampert R, Weih EH, Breucking E, Kirchhoff S, Lazica B, Lang $\mathrm{K}$. [Postoperative bilateral compartment syndrome resulting from prolonged urological surgery in lithotomy position. Serum creatine kinase activity (CK) as a warning signal in sedated, artificially respirated patients]. Anaesthesist 1995; 44(1): 43-7.

[51] Hargens AR, Schmidt DA, Evans KL, Gonsalves MR, Cologne JB, Garfin SR, Mubarak SJ, Hagan PL, Akeson WH. Quantitation of skeletal-muscle necrosis in a model compartment syndrome. J Bone Joint Surg Am 1981; 63(4): 631-6.

(C) Lasanianos et al.; Licensee Bentham Open.

This is an open access article licensed under the terms of the Creative Commons Attribution Non-Commercial License (http://creativecommons.org/licenses/by-nc/3.0/) which permits unrestricted, non-commercial use, distribution and reproduction in any medium, provided the work is properly cited. 